\title{
High-power, photofission-inducing bremsstrahlung source for intense pulsed active detection of fissile material
}

\author{
J. C. Zier, ${ }^{1}$ D. Mosher, ${ }^{2}$ R. J. Allen, ${ }^{1}$ R. J. Commisso, ${ }^{1}$ G. Cooperstein, ${ }^{2}$ D. D. Hinshelwood, ${ }^{1}$ \\ S. L. Jackson, ${ }^{1}$ D. P. Murphy, ${ }^{1}$ P. F. Ottinger, ${ }^{2}$ A. S. Richardson, ${ }^{1}$ J. W. Schumer, ${ }^{1}$ \\ S. B. Swanekamp, ${ }^{1}$ and B. V. Weber ${ }^{1}$ \\ ${ }^{1}$ Naval Research Laboratory, Plasma Physics Division, Washington, DC 20375, USA \\ ${ }^{2}$ Engility Corporation, Independent contractors, Chantilly, Virginia 20151, USA
}

(Received 25 September 2013; published 13 June 2014)

\begin{abstract}
Intense pulsed active detection (IPAD) is a promising technique for detecting fissile material to prevent the proliferation of special nuclear materials. With IPAD, fissions are induced in a brief, intense radiation burst and the resulting gamma ray or neutron signals are acquired during a short period of elevated signalto-noise ratio. The $8 \mathrm{MV}, 200 \mathrm{kA}$ Mercury pulsed-power generator at the Naval Research Laboratory coupled to a high-power vacuum diode produces an intense $30 \mathrm{~ns}$ bremsstrahlung beam to study this approach. The work presented here reports on Mercury experiments designed to maximize the photofission yield in a depleted-uranium (DU) object in the bremsstrahlung far field by varying the anode-cathode (AK) diode gap spacing and by adding an inner-diameter-reducing insert in the outer conductor wall. An extensive suite of diagnostics was fielded to measure the bremsstrahlung beam and DU fission yield as functions of diode geometry. Delayed fission neutrons from the DU proved to be a valuable diagnostic for measuring bremsstrahlung photons above $5 \mathrm{MeV}$. The measurements are in broad agreement with particlein-cell and Monte Carlo simulations of electron dynamics and radiation transport. These show that with increasing AK gap, electron losses to the insert and outer conductor wall increase and that the electron angles impacting the bremsstrahlung converter approach normal incidence. The diode conditions for maximum fission yield occur when the gap is large enough to produce electron angles close to normal, yet small enough to limit electron losses.
\end{abstract}

DOI: 10.1103/PhysRevSTAB.17.060401

PACS numbers: 52.59.-f, 52.65.Rr, 25.85.Jg, 03.50.-z

\section{INTRODUCTION}

Detection of fissile material is important for preventing the proliferation of special nuclear materials and nuclear weapons. Active detection methods utilize a probing source of photons, neutrons, or charged particles to induce fissions inside fissile material, temporarily elevating the gamma ray and neutron emission rates above the natural rates accessible to passive detection methods [1]. This elevation improves the detection capability of a given fission detection instrument by increasing the signal magnitude. It can also improve the probability of detection by adding a higher energy spectral component to the signal, such as inducing a delayed gamma ray component that extends to several $\mathrm{MeV}$ on top of the weakly penetrating $186 \mathrm{keV}$ natural gamma ray line from ${ }^{235} \mathrm{U}$. One active detection technique that maximizes the signal-to-background ratio (SBR) is intense pulsed active detection (IPAD). This technique induces the required fissions for detection using a brief, intense radiation burst and acquires the resulting gamma ray or

Published by the American Physical Society under the terms of the Creative Commons Attribution 3.0 License. Further distribution of this work must maintain attribution to the author $(s)$ and the published article's title, journal citation, and DOI. neutron signal during the subsequent short period of maximum elevated SBR. The reduction in the required counting time reduces the passive background contribution in the measurement compared with traditional (i.e., continuous-wave) active detection sources [2]. This improvement in SBR from the IPAD technique therefore permits reduced dose, increased range, or increased shielding penetration for given detection criteria when the induced background is properly mitigated [3-7]. In order to induce this large burst of fissions for detection, IPAD requires an intense source of probing radiation.

One option for producing a probing source suitable for IPAD is to use an intense bremsstrahlung beam created in a high-power electron-beam diode coupled to a pulsed-power generator. Pulsed-power generators, such as the Mercury inductive voltage adder (IVA) at the Naval Research Laboratory [8], store hundreds of kilojoules to megajoules in capacitors and are capable of releasing that energy in tens of nanoseconds to produce TW-scale, multi-MeV ion or electron beams. For this experimental campaign, the Mercury generator was operated in negative polarity at $-8 \mathrm{MV}[8,9]$ to accelerate a $200-\mathrm{kA}$ electron beam into a Ta-based bremsstrahlung converter package. The diode geometry, described in detail in Sec. II A, was designed for multishot operation and the anode-cathode (AK) gap was varied to optimize the photofission yield in a depleted 
uranium (DU) object on the diode axis of symmetry in the bremsstrahlung far field.

This work is a significant expansion in analysis detail beyond the preliminary findings reported in Ref. [10]. An extensive suite of diagnostics was fielded to measure the bremsstrahlung beam parameters, both in terms of dose distributions and fission yield, as functions of diode geometry. These diagnostics are described in Sec. II B and are complemented by the diode electrical diagnostics. The experiment was modeled in detail, from the electrical power flow entering the diode to the emitted $\mathrm{x}$ rays, using the simulation codes LSP [11] and ITS [12]. Details for these simulations are given in Sec. II C, and the various measurement results are compared to modeling in Sec. III. The work concludes with a summary and a discussion of ongoing and future research in Sec. IV.

\section{EXPERIMENTAL CONFIGURATION}

This work builds on previous studies of IVA-driven, high-power electron beam diodes for flash x-ray radiography [13-16] and bremsstrahlung production, particularly an extensive series of experiments using the Hermes III generator at Sandia National Laboratories [17-21]. Our objectives have two significant differences from that previous work. First, rather than maximizing the radiation dose, we wish to maximize photofissions, which require photon energies in excess of $5 \mathrm{MeV}$, while minimizing an unnecessary dose [22]. Second, we are interested in the far field. These important differences led to a thinner x-ray converter package optimized for high-energy-photon production rather than maximum dose, and to an emphasis on near-normal electron impact onto the converter at peak voltage to benefit from the $1 / \gamma$ Lorentz factor directionality of the bremsstrahlung intensity field.

Our diode development was guided by two important constraints. First, we will ultimately need to collimate the bremsstrahlung radiation to minimize the collateral dose. This drives us toward minimizing the diode radius. Second, the anode must remain intact after the pulse, which requires that the electron beam not pinch. This necessitates a large electron-beam radius so that the electron heating of the anode does not produce anode plasma [23], which leads to intense ion flow and electron pinching [24].

\section{A. High-power diode and electrical diagnostics}

The diode geometry for this experiment (Fig. 1) consisted of a cylindrically symmetric coaxial magnetically insulated transmission line (MITL) [25] that delivered power into the diode region. The MITL outer conductor inner radius was $19.8 \mathrm{~cm}$, and the cathode center conductor radius was $8.4 \mathrm{~cm}$ (51- $\Omega$ vacuum impedance). The $\mathrm{AK}$ vacuum-gap distance was measured between the hemispherical end of the Mercury center conductor and the

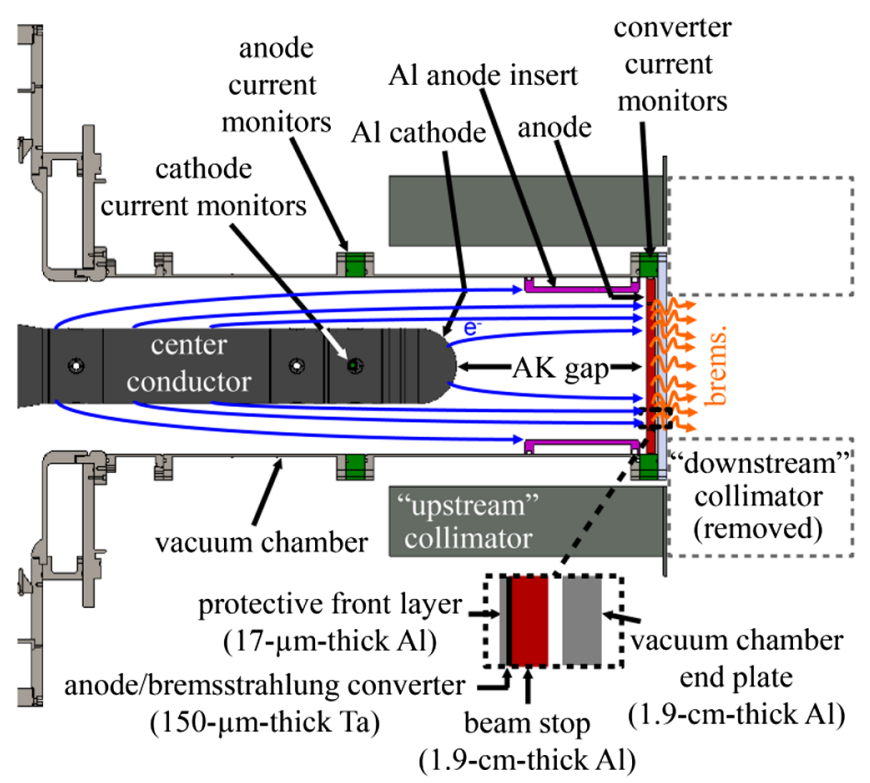

FIG. 1. Diode region geometry and electrical diagnostics for this experiment. The MITL feeds the diode from the left. An additional "downstream" collimator (shown as dashed lines) was not fielded during this experiment but served as a constraint on the useful electron beam radius.

converter package mounted at the end of the grounded outer conductor anode. This gap spacing was varied between 23 and $43 \mathrm{~cm}$ to determine the changes in electron beam dynamics and the resulting bremsstrahlung emission. The hemisphere was made of aluminum with a bare metallic surface. Bare metal was found to improve the shot-to-shot reproducibility for consecutive shots without breaking vacuum, and the hemisphere was chosen to provide a more uniform and paraxial electron beam compared with a more traditional thin-walled cylinder, as described in Ref. [22]. Upstream of this hemisphere, the center conductor was coated with a thin layer of carbon for more uniform electron emission and power flow in the MITL. The converter package consisted of a $17-\mu \mathrm{m}$-thick $\mathrm{Al}$ layer, a $150-\mu \mathrm{m}$-thick Ta layer, a 1.9-cm-thick $\mathrm{Al}$ beam stop, and a $1.9-\mathrm{cm}$-thick $\mathrm{Al}$ vacuum-air-interface flange (endplate). The Ta served as the $\mathrm{x}$-ray converter and the initial Al layer suppressed plasma formation and ion emission, which would have resulted in beam pinching and damage to the converter. The $150-\mu \mathrm{m}$-thick Ta layer used for this experiment was approximately 3 times thinner than a traditional, dose-optimized converter [22]. Current in the diode region was measured at three locations (Fig. 1). Anode current (B-dot monitors) monitors measured the total current entering the diode region upstream of the cathode hemisphere. Along with these anode monitors, cathode monitors at nearby axial locations were used to determine the diode voltage using the Mendel method for a MITL [26]. Additional B dots were fielded just upstream of the endplate to measure the beam current that struck the 
converter; the difference between the total and converter currents is the current lost to the outer conductor.

For diode gaps of 23 to $43 \mathrm{~cm}$, the diode impedance is roughly 3 to 5 times the self-limited flow (SLF) impedance of the MITL $[26,27]$. For diode impedance larger than the SLF impedance, the MITL in the IVA architecture of Mercury limits the effective load impedance to the 40-Ohm SLF impedance, independent of AK gap $[28,29]$. What does change with gap size is the vacuum electron flow in the MITL and diode. As the gap increases, the electron beam moves radially outward and impacts the converter at larger radius, increasing losses to the outer conductor, and causing the beam to strike the converter closer to normal incidence.

Although not present for any of the diode development experiments reported here, an additional thick steel collimator, shown as dashed lines in Fig. 1, was often employed during active detection experiments that used this bremsstrahlung source. This collimator had an inner radius of $16.5 \mathrm{~cm}$, so that any radiation produced on the converter at radii greater than $16.5 \mathrm{~cm}$ would have been severely attenuated. In order to push the electron beam impact zone radially inward so that the majority of the forward-going bremsstrahlung photons would clear this external aperture, a $25-\mathrm{cm}$-long aluminum insert with a $16.5 \mathrm{~cm}$ inner radius was installed to create an indented anode geometry (Fig. 1) [30]. This insert was installed for AK gaps of $35 \mathrm{~cm}$ and greater because smaller AK gaps already satisfied this collimation condition.

\section{B. Bremsstrahlung diagnostic geometry}

An array of diagnostics was fielded outside the vacuum system to measure the bremsstrahlung spectrum as a function of converter radius, angle, and energy. A DU plate served as an activation diagnostic for measuring the high-energy portion of the bremsstrahlung spectrum. The plate consisted of a $99.8 \%$ U-238, $0.2 \%$ U-235 slab with $30.48 \mathrm{~cm}$ square faces and $2.54 \mathrm{~cm}$ thick, encased within an aluminum jacket $0.32 \mathrm{~cm}$ thick on the slab faces and $1.27 \mathrm{~cm}$ thick on the slab edges. Photons with energies above $5 \mathrm{MeV}$ induced photofission events in the DU [31] that were measured by a He-3 delayed neutron detector (see the "low-E" build in [32]). The time-integrated x-ray dose was measured using $\mathrm{CaF}_{2}$ thermoluminescent dosimeters (TLDs) encased within 4-mm-thick walled aluminum equilibrators. The TLDs primarily measure the intensity of low-energy photons in the 8-MeV-endpoint bremsstrahlung spectrum. These TLDs were individually calibrated using a Co-60 source, resulting in about a 3\% instrumentation uncertainty in the measured dose [33]. A pinhole camera (PHC) was used to measure the time-integrated spatial distribution of $\mathrm{x}$-ray emission on the converter to diagnose the impacting electron beam profile. The PHC itself consisted of a thick lead housing with a 1-mmdiameter tungsten pinhole, providing 0.12 magnification onto a Fujifilm high-sensitivity image plate. The imaging resolution for the PHC was about $1 \mathrm{~cm}$ on the converter. An array of lead-collimated scintillator/photodiodes was used to monitor the time-dependent bremsstrahlung beam dynamics. The time-integrated response for these detectors was directly proportional to the dose measured by the TLDs [33].

Two major diagnostic configurations were used: an onaxis DU plate setup and an on-axis pinhole camera setup. These configurations are shown in Figs. 2(a) and 2(b). The DU and PHC could not be fielded on axis simultaneously without blocking each other, so when obtaining PHC data, the DU was moved $15^{\circ}$ off axis to measure the highenergy spectral component at a different angle. Near-field dose measurements were made using TLDs mounted onto the outside face of the $\mathrm{Al}$ vacuum endplate $3.8 \mathrm{~cm}$ from the Ta. These endplate dose measurements provided an absolutely calibrated measure of the time-integrated electron beam profile on the converter, but with cruder spatial resolution than the PHC measurements.

The on-axis DU configuration featured the DU plate at $5 \mathrm{~m}$ from the converter with the DU face tilted $45^{\circ}$ from the beam axis. A He-3 detector was positioned $1 \mathrm{~m}$ from the DU face exposed to the bremsstrahlung beam to measure delayed fission neutrons emitted by the DU. A photodiode array was positioned $3.5 \mathrm{~m}$ from the converter at $5^{\circ}, 10^{\circ}, 20^{\circ}$, and $30^{\circ}$ from the beam axis. An angular TLD array ( $\varphi$ map) was positioned $3 \mathrm{~m}$ from the converter center at $\pm 5^{\circ}, \pm 10^{\circ},+15^{\circ}, \pm 20^{\circ}, \pm 30^{\circ}$, and $+45^{\circ}$. TLDs were positioned on axis at $1,2,3$, and $4 \mathrm{~m}$ from the converter ( $\mathrm{Z}$ map). A TLD pair was placed on the exposed DU face at $5 \mathrm{~m}$ and $0^{\circ}$. The endplate TLD map for the on-axis configuration was designed to provide the best resolution near the predicted radius for the annular electron beam. Endplate TLDs were positioned at 10-, 14-, and $16.5 \mathrm{~cm}$ radii from the beam axis at $0^{\circ}, 90^{\circ}, 180^{\circ}$, and $270^{\circ}$ in azimuth around the beam axis. Additional TLDs were positioned on a $19 \mathrm{~cm}$ radius at $0^{\circ}$ and $180^{\circ}$, and a TLD pair was positioned on the beam axis. For roughly half of the shots, additional TLDs were added on a $14 \mathrm{~cm}$ radius at $45^{\circ}, 135^{\circ}, 225^{\circ}$, and $315^{\circ}$.

The PHC configuration featured the PHC diagnostic positioned on the beam axis at $4 \mathrm{~m}$ from the converter. For this setup, the DU plate was moved to $-15^{\circ}$ with the same $5 \mathrm{~m}$ distance to the converter. The plate's $45^{\circ}$ rotation to the bremsstrahlung beam was maintained at the new location. The He-3 detector was not moved between configurations, so the detector location relative to the repositioned DU plate was $84 \mathrm{~cm}$ away and $15^{\circ}$ from the DU plate's surface normal. The photodiode array was identical to the on-axis DU configuration. The number of TLDs was reduced for logistical reasons to $\pm 10^{\circ}$, and $+30^{\circ}$ at $3 \mathrm{~m}$ for the $\varphi$ map, and to 1,2 , and $3 \mathrm{~m}$ at $0^{\circ}$ for the $\mathrm{Z}$ map. One TLD was also placed on the DU face at $5 \mathrm{~m}$ and $-15^{\circ}$. 

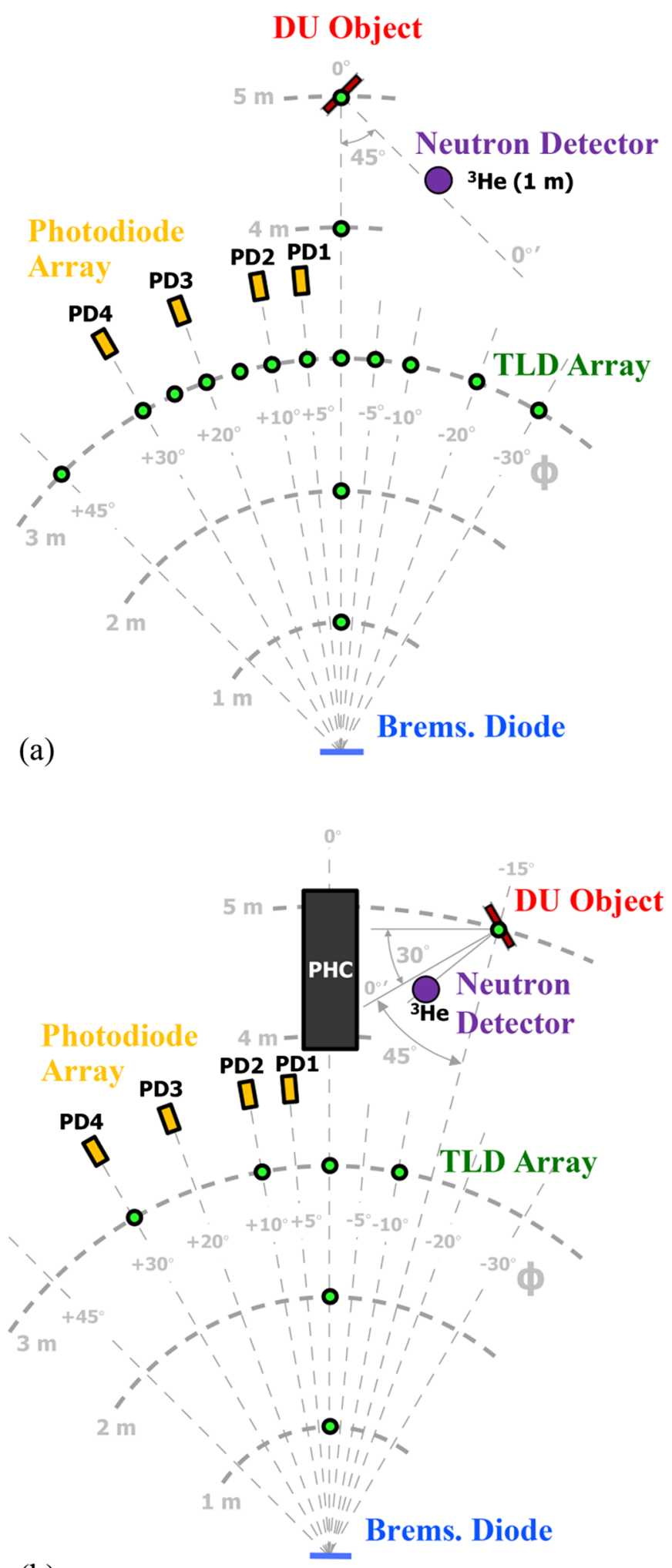

(b)

FIG. 2. Experimental configurations for (a) the on-axis DU and (b) the on-axis PHC diagnostics. Thermoluminescent dosimeters are shown as small circles and the He-3 delayed neutron detector is shown as a larger circle. The photodiode array is represented by small rectangles and was located along the $3.5 \mathrm{~m}$ radius from the converter. Both (a) and (b) are not precisely to scale.

\section{Simulation methods}

Detailed modeling of the experiments was performed using the particle-in-cell (PIC) code LSP [11] to compute the electromagnetic fields and electron-beam dynamics in the diode region. Among the LSP outputs were electron and photon particle lists that were imported into the ITS Monte Carlo radiation-transport codes [12] for further processing in order to model the responses of the deployed radiation diagnostics.

PIC simulations with LSP were performed for a range of anode-cathode gaps, both with and without the anode insert, and included the last section of the MITL. These 2D r-z, cylindrically symmetric simulations were electrically driven by a forward-going 8.35-MV-peak voltage waveform launched at the upstream boundary of the simulation approximately $130 \mathrm{~cm}$ upstream from the converter. This voltage waveform was taken from a representative full-system LSP simulation of an open-circuit load driven by forward-going waves from experimental current and voltages at the upstream pulse-forming-line [9]. The diode gap was varied between 23 and $43 \mathrm{~cm}$ by changing the length of the MITL section. Space-charge-limited electron emission from the center conductor was allowed with a threshold of $200 \mathrm{kV} / \mathrm{cm}$ in order to model vacuum electron flow in the MITL and emission from the hemispherical cathode. The total current flowing in the simulated diode was measured by LSP using a point detector located on the outer conductor about $48 \mathrm{~cm}$ upstream from the vacuum endplate. This point is about $10 \mathrm{~cm}$ upstream of the anode current monitors in the experiment. Another point detector just upstream of the endplate measures the current impacting the converter (Fig. 1). LSP grid spacing of $\Delta r=\Delta z \approx 1 \mathrm{~mm}$ was used. For these simulations, the Courant limit $(\Delta t \leq \Delta x / c$, where $c$ is the speed of light) sets the maximum time step at about 3 ps. To remain within this limit, a time step of $\Delta t \approx 2 \mathrm{ps}$ was used. The $\mathrm{Al}$ and $\mathrm{Ta}$ foils of the converter package were modeled in LSP using material layers with an axial thickness of a single cell $(1 \mathrm{~mm})$. Since this is larger than these actual foil thicknesses, artificially lowdensity materials were used in the simulation so that the correct total mass and axial column density was modeled.

One potential source of uncertainty in these simulations arises from our attempts to model the ion current flowing in the diode. The electron-beam fluence impacting the anode is far below the levels needed for space-charge-limited ion emission from thermal anode plasmas that occurs in pinched-beam diodes (corroborated by the observed absence of pinching). On the other hand, there is independent experimental evidence for relatively small ion currents generated by electron-stimulated desorption [23,34]. This presence of small ion currents was also verified in previous Mercury experiments using nuclear activation [22]. However, these measurements showed that the ion current was very spotty (and thus inherently 3D). 
We find that in order to reproduce the experimental results in modeling, small ion currents in the load region are required. Ion flow is modeled in our 2D LSP simulations by ion creation with a prescribed probability for each electron striking the anode. LSP simulations have been performed using stimulation probabilities in the range of $0 \%-0.6 \%$. Comparison with earlier $23 \mathrm{~cm}$ gap experiments indicated good agreement for $0.2 \%-0.4 \%$ ions [35]. All of the LSP/ ITS results presented here for different gaps assume $0.2 \%$ stimulated emission of ions.

\section{MEASUREMENT AND SIMULATION RESULTS}

\section{A. Electron beam expansion and current losses}

Figure 3(a) shows the measured current in the diode from the anode monitors and the current impacting the converter for four sequential $40 \mathrm{~cm}$ gap shots (thin curves) compared to LSP predictions (heavier, noisier curves). Figure 3(b) shows LSP $r B_{\theta}$ map snapshots (current enclosed within radius $r$ ) in the diode at 3 times during the current history of Fig. 3(a). The overlaid current contour increments are $10 \%$ of the peak current.

The predicted converter current is seen to rise earlier than the measurements in Fig. 3(a), though its peak value and fall are in good agreement. Figure 3(b) shows that the delay in the rise and early decay of the converter current are explained by enhanced scrape-off of electrons by the insert at times away from peak current due to reduced magnetic confinement of the beam. It is expected that the later rise of the measured converter current is due to $3 \mathrm{D}$ asymmetries and instabilities that further reduce the experimental beam confinement and are not modeled in 2D LSP. The additional electron charge reaching the converter in LSP will result in predicted doses that are higher than measured.

Figure 4 shows LSP current contours in the diode for various diode gaps at the time of peak diode voltage [50 ns in Fig. 3(a)]. The figure shows the progression of the beam to larger radius as the gap increases and an accompanying reduction in converter current. Note that the electron impact angles on the converter are closer to normal for the larger gaps. The trade-off between loss current and impact angle is crucial for far-field fission optimization. Although electrons in the loss current produce $\mathrm{x}$ rays as they impact the outer conductor, they do not strike high-atomic-number material in a favorable geometry, and they do not efficiently produce high-energy $\mathrm{x}$ rays for inducing photofission on axis in the far field.

For one of the shots in Fig. 3(a), the expected x-ray production from the converter current $\left(I_{\text {con }}\right)$ was checked against the $\mathrm{x}$ rays measured with the photodiode at $5^{\circ}$ using an $I \times V_{\text {diode }}^{2.8}$ scaling [36] as shown in Fig. 5. The same scaling was used to predict the $\mathrm{x}$ ray production using the total diode current $\left(I_{\text {diode }}\right)$. The converter-current scaling was an excellent match to the photodiode signal shape, a
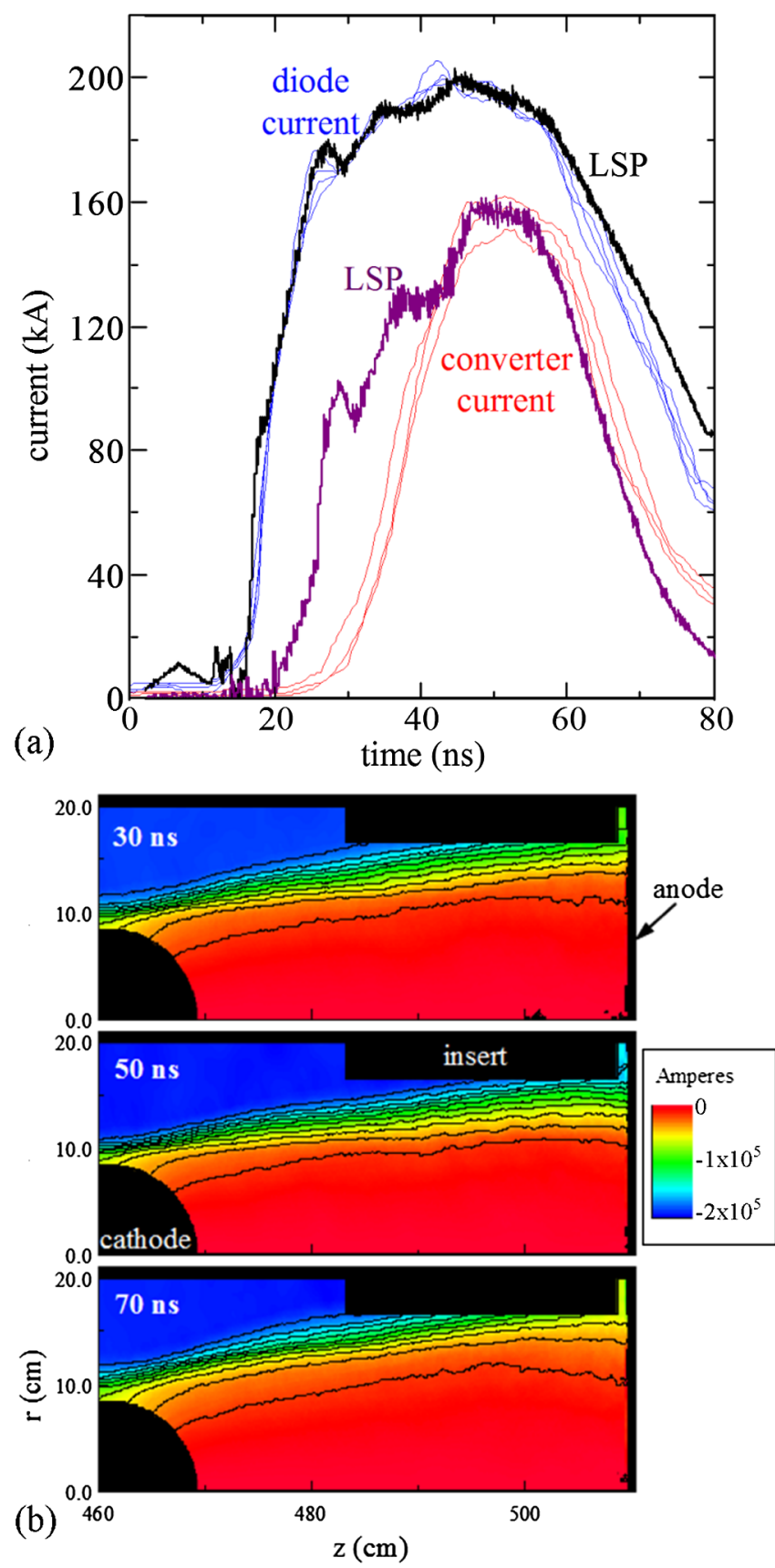

FIG. 3. (a) Electron current in the diode and on the converter for four sequential $40 \mathrm{~cm}$ gap shots (thin curves) compared to LSP predictions (heavier, noisy curves). (b) LSP $r B_{\theta}$ distributions (current enclosed within radius $r$ ) in the diode at 3 times (dashed lines in Fig. 3a) for a $40 \mathrm{~cm}$ gap. Overlaid current contours are in $10 \%$ increments.

strong indication that the converter B-dot monitors provided an accurate measurement of the current producing the $\mathrm{x}$ rays. Four-shot averages of these converter-current measurements are shown in Fig. 6(a) for the 35, 40, and $43 \mathrm{~cm}$ AK gap configurations (the converter B-dot monitors did not function for AK gaps below $35 \mathrm{~cm}$ because the insert was not present to shield them from the electron 


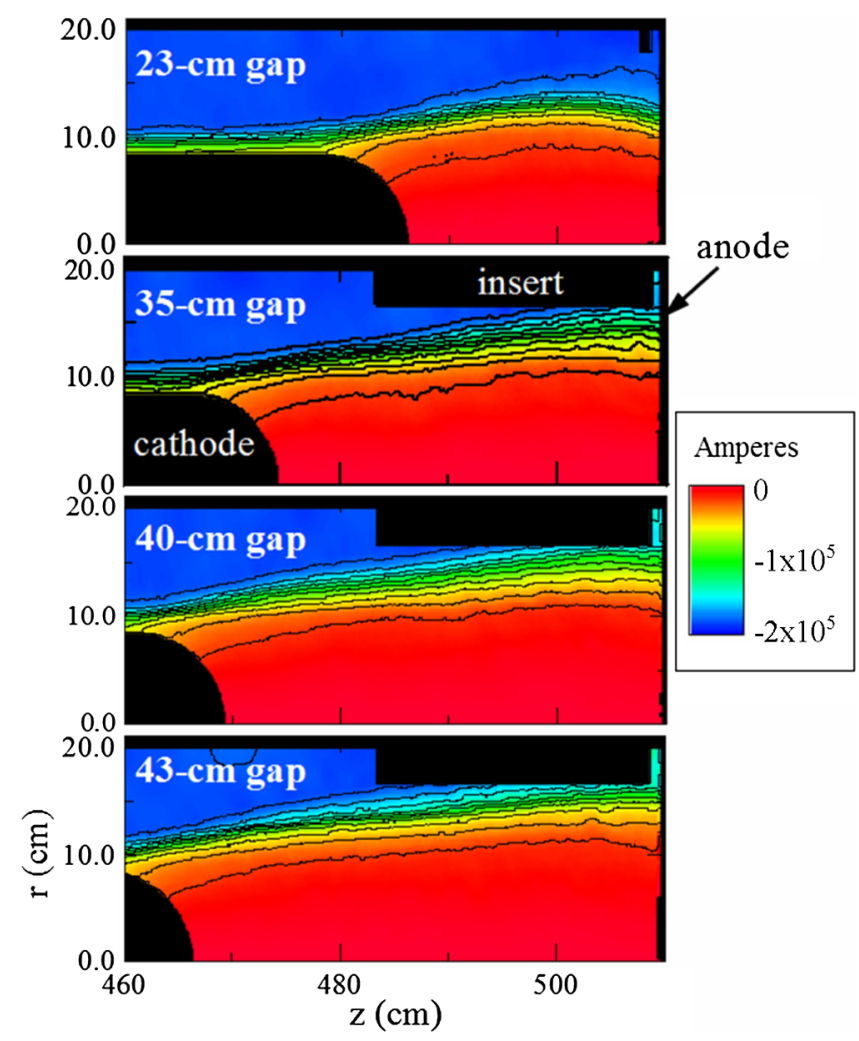

FIG. 4. LSP $r B_{\theta}$ distributions (current enclosed within radius $r$ ) in the diode for various diode gaps at the time of peak diode voltage (50 ns in Fig. 3a). Overlaid are current contours in $10 \%$ increments.

beam). The converter current for the $35 \mathrm{~cm}$ AK gap configuration reached the total diode current approximately $20 \mathrm{~ns}$ into the pulse. These currents agreed for another $20 \mathrm{~ns}$ before the converter current began to drop more quickly

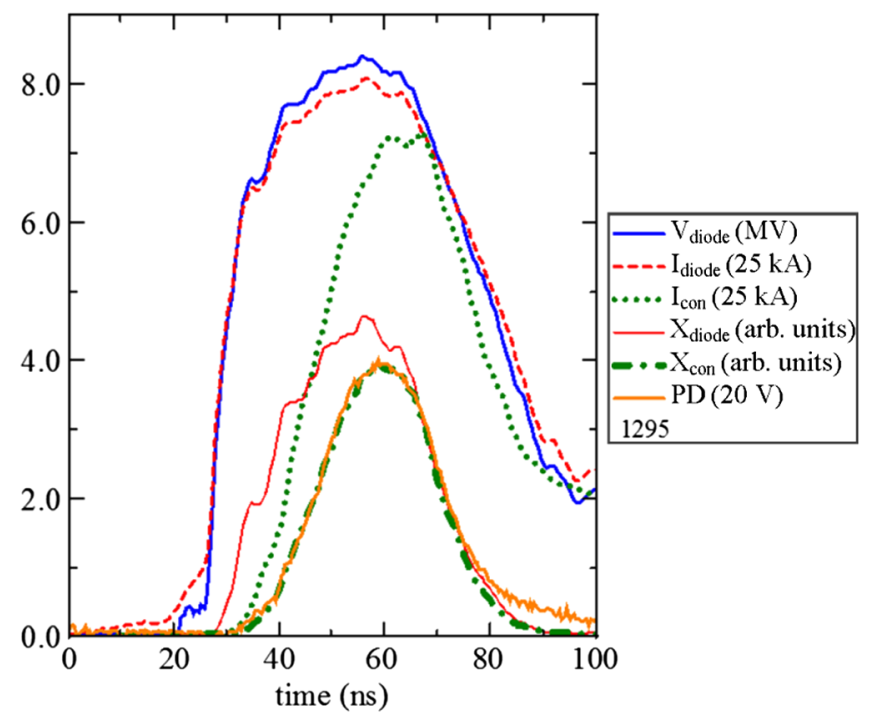

FIG. 5. Electrical traces for shot 1295 with a $40 \mathrm{~cm}$ gap. $I_{\text {diode }}$ is the total current entering the diode region, $I_{\text {con }}$ is the current impacting the converter, $X_{\text {diode }}$ is $I_{\text {diode }} V^{2.8}, X_{\text {con }}$ is $I_{\text {con }} V^{2.8}$, and $\mathrm{PD}$ is the signal from the photodiode $5^{\circ}$ off the axis of symmetry. than the diode current. For the larger 40 and $43 \mathrm{~cm}$ AK gap configurations, the converter current never reached the total diode current; the peak current losses were approximately $25 \%$ and $37.5 \%$, respectively. Figure 6(b) shows the LSPpredicted currents corresponding to the measurements in Fig. 6(a). These again show increasing current loss with increasing gap.

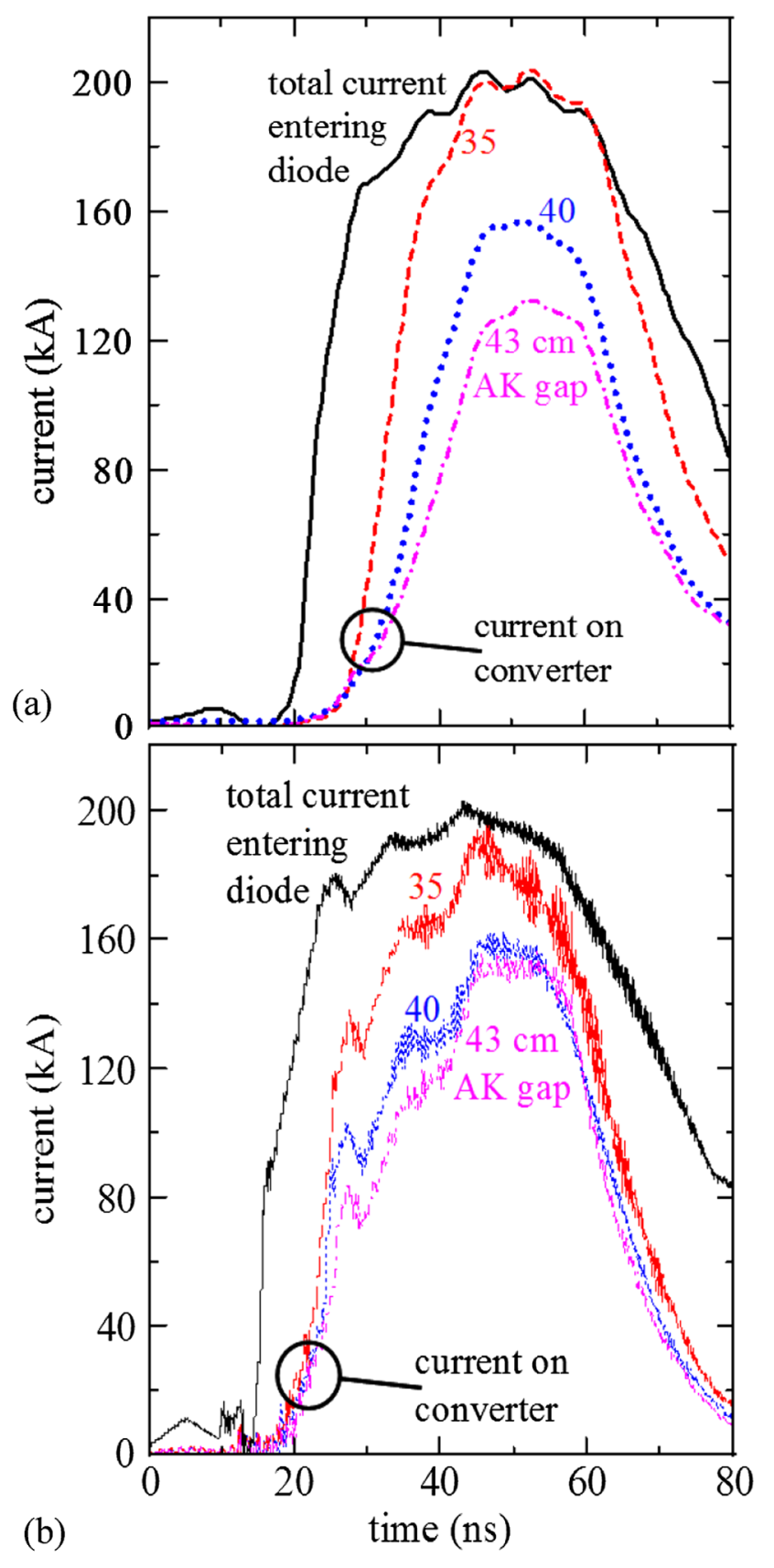

FIG. 6. (a) Electron current on the converter for 35, 40, and $43 \mathrm{~cm}$ AK gaps compared to that entering the diode region. Traces are 4 shot averages for each gap. (b) LSP electron currents on the converter for 35,40 , and $43 \mathrm{~cm}$ AK gaps compared to that entering the diode region. 
The measured beam-current losses support the LSP results that time-dependent radial expansion of the annular electron beam into the anode insert reduces the converter current, and that the losses increase with AK gap. This explanation for the current loss was confirmed using the PHC. Representative PHC images in Fig. 7 show that the annular beam strikes the converter at larger radii when the gap is increased, and that bremsstrahlung production is weak outside of the $16.5 \mathrm{~cm}$ insert radius. The integrated image plate intensities were scaled to the TLD dose measurements from the corresponding shot to achieve a relative intensity calibration between the images. These time-integrated measurements show the electron beam deposition was approximately azimuthally symmetric about the beam axis, although there may be some fine azimuthal structure visible on some shots. A more detailed investigation into any azimuthal symmetry is a topic of future research and will require time-resolved PHC imaging.

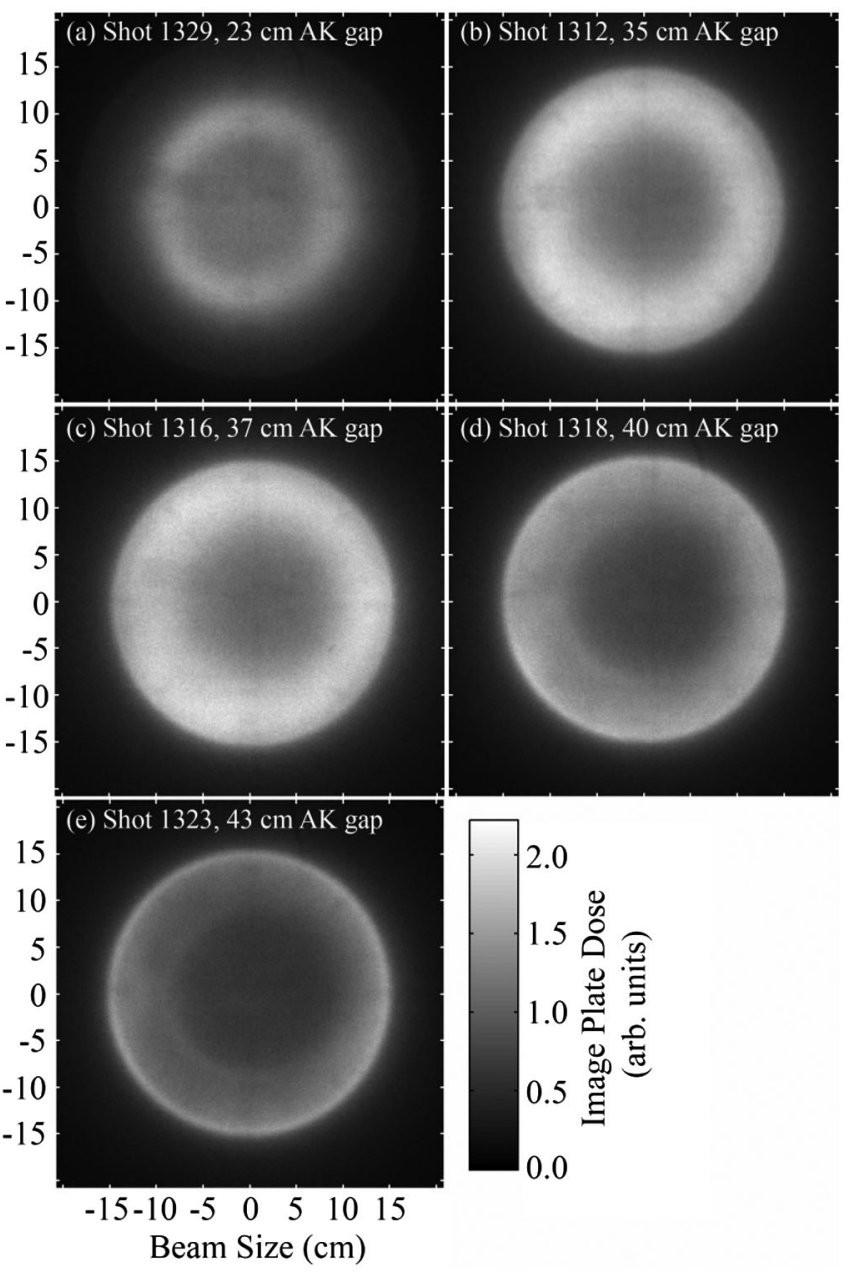

FIG. 7. Representative pinhole camera images for the 23, 35, 37, 40, and $43 \mathrm{~cm}$ AK gaps are shown in (a)-(e), respectively. The integrated image plate intensities were scaled to the TLD dose measurement at $1 \mathrm{~m}$ to achieve a relative intensity calibration between the images shown.
For a quantitative comparison between these PHC images, radial profiles were generated by azimuthally averaging the image plate intensities. These radial profiles are plotted in Fig. 8(a) and the predicted radial profiles from
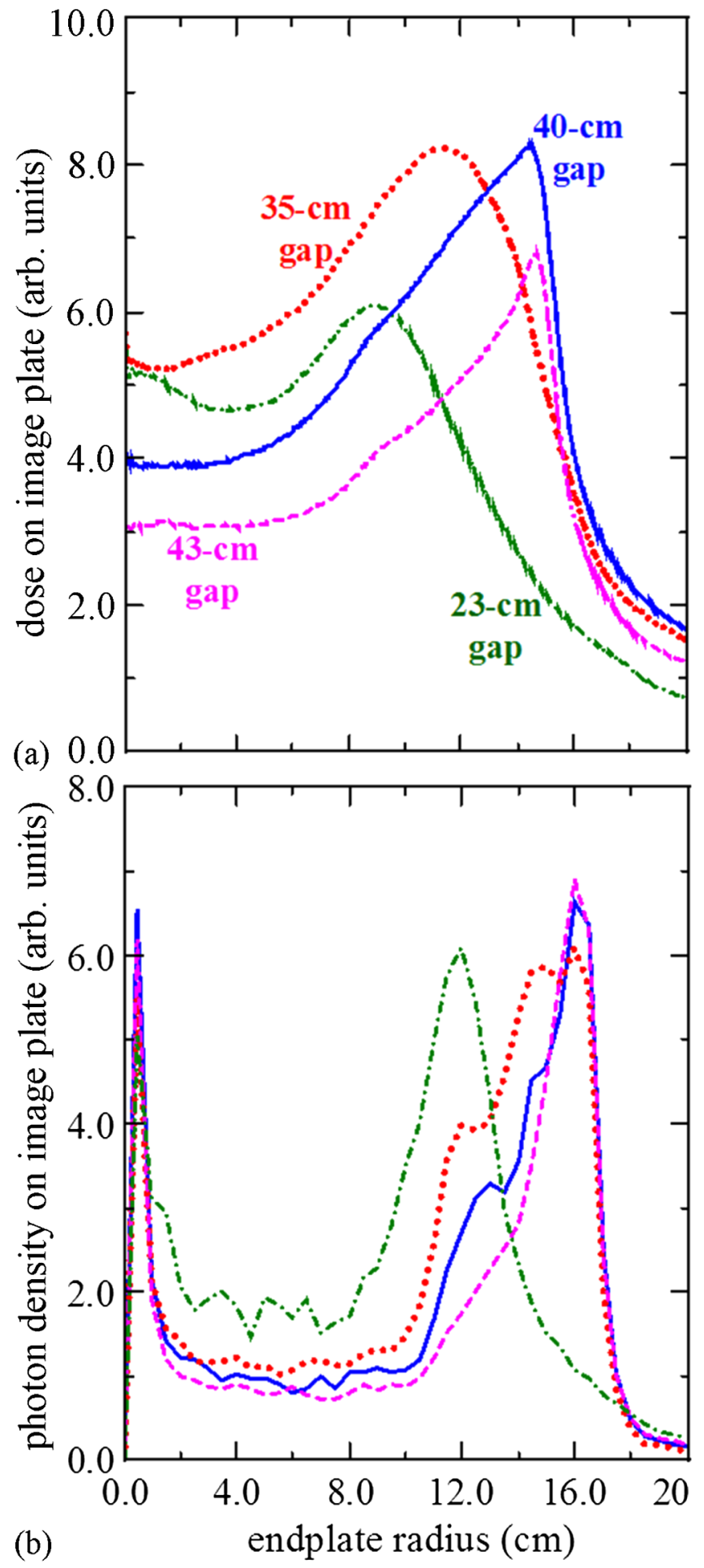

FIG. 8. (a) Radial distribution of dose on the endplate inferred from the pinhole-camera image-plate intensity for different AK gaps. (b) Radial distribution of x-ray intensity on the endplate from LSP/ ITS simulations of different AK gaps. Curve labels are as in Fig. 8(a). 
LSP/ITS are plotted in Fig. 8(b). Image plate dimensions have been converted to those on the endplate using the 0.12 PHC magnification. The predicted radial profiles from LSP/ITS qualitatively show the same patterns as the measurements for radii greater than $2 \mathrm{~cm}$. The simulations substantially overpredict the beam radius for the 23 and $35 \mathrm{~cm}$ gaps, and show a deeper depression at smaller radii for all gaps. This suggests an incomplete model for ion emission, and/or an electron-emission threshold that is too high from the cathode hemisphere. Additionally, the LSP/ ITS model underpredicts electron beam losses to the anode insert for the $43 \mathrm{~cm}$ gap, which is consistent with the above indication of enhanced experimental losses due to 3D effects (expected to be most pronounced close to the anode surface, and therefore with the $43 \mathrm{~cm}$ gap).

Near the beam axis, the simulations contained an artifact consisting of a sharp spike in photon emission that has not been seen in any x-ray pinhole images or near-field dose maps. This artifact is a result of the $2 \mathrm{D}$ nature of the simulation. Cold ions, with zero angular velocity when emitted from the anode, are accelerated radially inward towards the symmetry axis. Those that cross the axis in the AK gap create a channel of artificially high ion density which draws in matching-density electrons responsible for the bremsstrahlung spike seen in Fig. 8(b). However, the artifact has little effect on predicted far-field dose and fission yields because of the small on-axis volume associated with emission from the spike. Ongoing LSP simulations show that introducing a $30 \mathrm{keV}$ transverse temperature on the ions at emission is sufficient to flatten

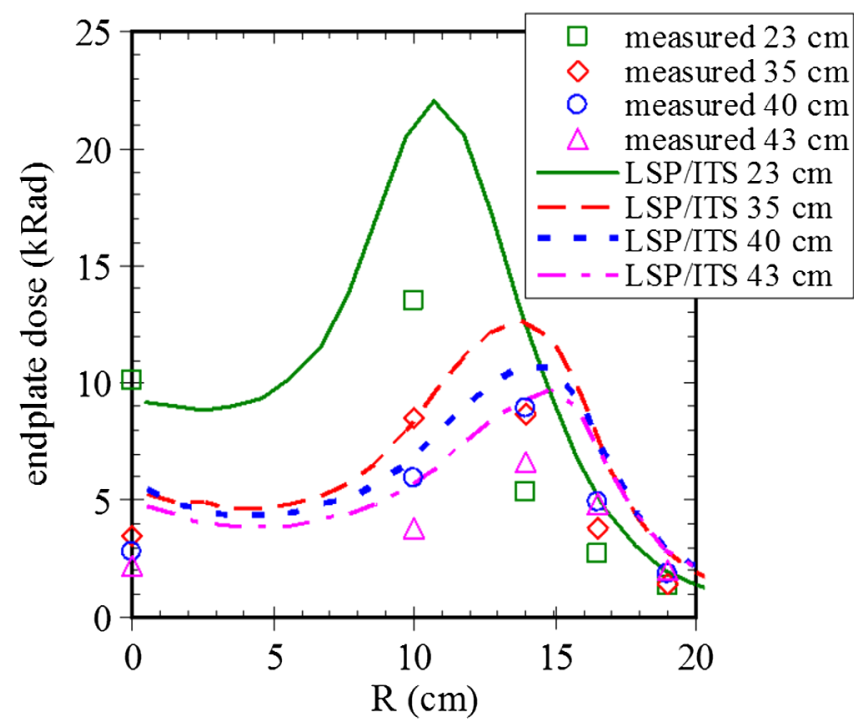

FIG. 9. Radial variation of endplate dose for different AK gaps. For the measured points, the four azimuthally distributed TLDs at each radius are averaged for each shot. These azimuthal averages are then averaged for 4 to 6 shots at each AK gap. The measured points have shot-to-shot variations of about $\pm 10 \%$. the spike, but not to substantially raise photon emission inside the depression.

The near-field endplate TLD dose measurements, along with those simulated by LSP, are compared in Fig. 9. For the measured points, the four azimuthally distributed TLDs at each radius are averaged for each shot. The azimuthal averages are then averaged for $4-6$ shots at each AK gap. The measured points have shot-to-shot variations of azimuthally averaged dose of about $\pm 10 \%$. The doses from LSP/ITS are calculated from the radial distributions of photon spectral flux in 1-mm-thick $\mathrm{CaF}_{2}$ just outside the endplate. The absolute-value dose measurements appear consistent with corresponding pinhole images (Fig. 7) that are integrated over the solid angle associated with the several $\mathrm{cm}$ separating the outside of the endplate and the converter. The LSP/ITS simulations qualitatively matched the measurements, but with the expected higher dose from a higher simulated charge on the converter. Note that the axial artifact is not seen in the simulations as it is "blurred out" by the $3.8 \mathrm{~cm}$ distance separating the converter and dose-measurement plane.

\section{B. Bremsstrahlung angular distribution in the far field}

The time-integrated angular dose distribution was measured in the far field using the $\Phi$ map TLDs. These dose measurements were averaged over $4-6$ shots for a given diode configuration and the dose magnitudes were then scaled using $r^{-2}$ from the $3 \mathrm{~m}$ TLD locations to $1 \mathrm{~m}$. These measurements are shown in Fig. 10(a) for the 23, 35, 40, and $43 \mathrm{~cm}$ AK gap configurations. For the $23 \mathrm{~cm}$ gap, the on-axis dose at $1 \mathrm{~m}$ was $518 \mathrm{rad}\left(\mathrm{CaF}_{2}\right)$ with a FWHM $(2 \Phi)$ of $54^{\circ}$. This improved to $707 \mathrm{rad}$ and $36^{\circ}$ for the $35 \mathrm{~cm}$ gap, improved further to $731 \mathrm{rad}$ and $25^{\circ}$ for the $40 \mathrm{~cm}$ gap, and then degraded to $536 \mathrm{rad}$ and $31^{\circ}$ for the $43 \mathrm{~cm}$ gap. This clear increase in the on-axis dose, along with the narrowing in the angular dose distribution, indicated a more forwarddirected electron beam as the AK gap was increased from 23 to $40 \mathrm{~cm}$, and a reduction in beam quality for the $43 \mathrm{~cm}$ gap, along with a substantial penalty in dose. Additionally, the maximum shot-to-shot variation in the on-axis dose increased with AK gap: $\pm 4 \%$ at $23 \mathrm{~cm}, \pm 5 \%$ at $35 \mathrm{~cm}$, $\pm 8 \%$ at $40 \mathrm{~cm}$, and $\pm 13 \%$ at $43 \mathrm{~cm}$. We believe that the increase in shot-to-shot dose variation with increasing AK gap was caused by the electron beam grazing the anode insert, so that small changes in the beam radius introduce larger changes in dose from current scrape-off. For the largest gaps, where the beam was already partially impacting the anode insert, the effect was largest.

The LSP/ITS simulations of angular dose distribution shown in Fig. 10(b) capture the narrowing in angular dose with increasing gap, as well as the correct shapes for the dose distributions; however, the simulations overpredict the angular doses by approximately 30\% depending on the particular AK gap and angle. One potential source for the LSP/ITS overpredicting the dose has already been 


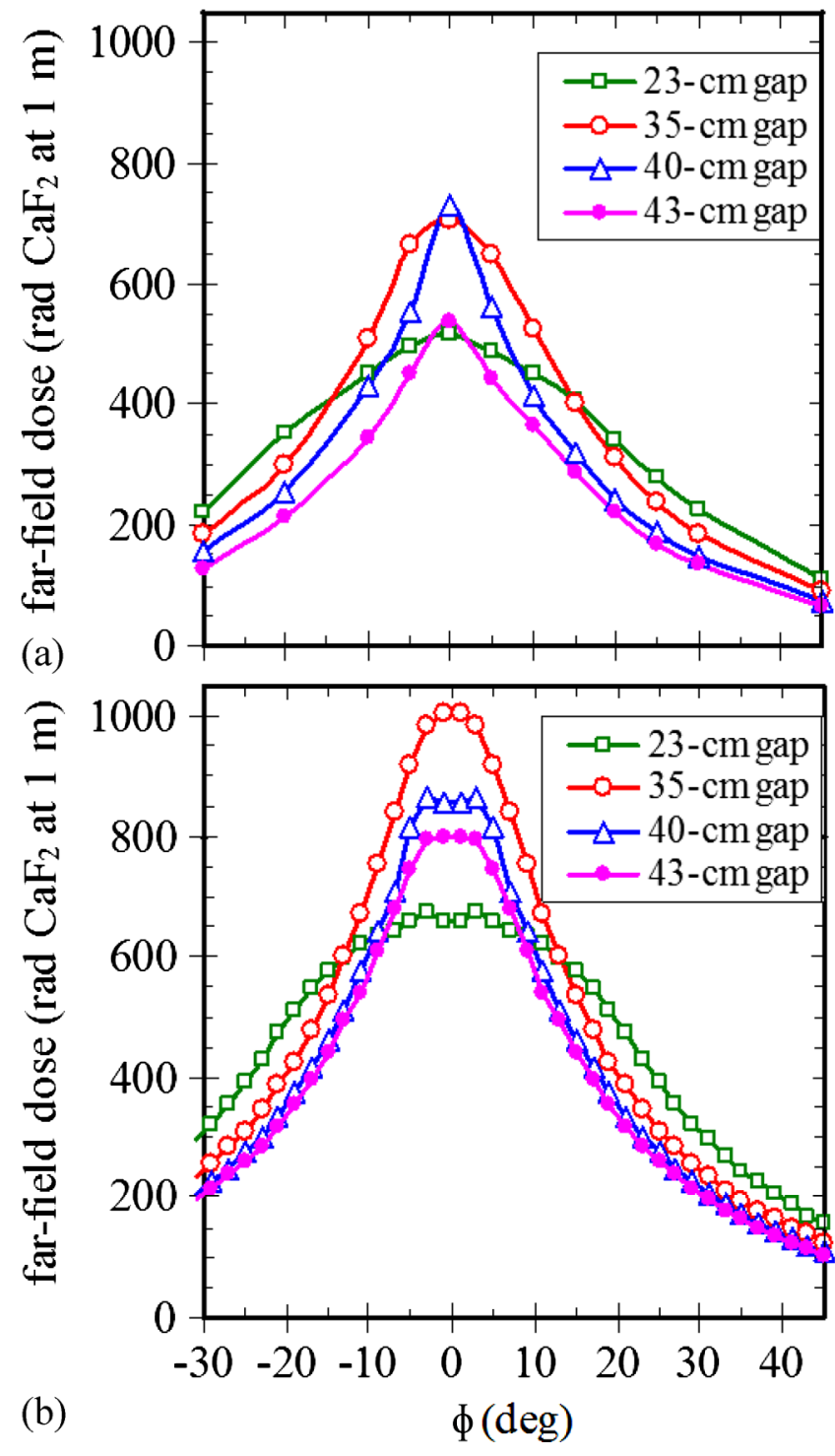

FIG. 10. (a) Measured time-integrated angular dose distributions from TLDs for various diode gaps. (b) LSP/ITS predictions of timeintegrated angular dose distributions for various diode gaps.

discussed with regard to Fig. 3(a) for a $40 \mathrm{~cm}$ gap where experimental current losses due to real-world threedimensional asymmetries and instabilities are not modeled by 2D LSP. This effect was also observed for other gaps in Fig. 6, especially for the $43 \mathrm{~cm}$ gap, where the predicted losses at peak voltage are substantially lower than measured. LSP simulations in 3D are planned to study the effects of nonuniform electron emission, ion formation and deposition, and geometrical deviations from azimuthal symmetry in more detail.

During the diode voltage pulse, the electron beam undergoes a time-dependent sweep from the outer conductor to the converter as the self-magnetic fields from the beam current bend the beam radially inward during the current rise, and then outward again during the current fall
(Fig. 3). The resulting time-dependent behavior of the angular dose was measured using the photodiode (PD) array and simulated using LSP/ITS as shown in Fig. 11(a) for the $23 \mathrm{~cm}$ AK gap, and in Fig. 11(b) for the $40 \mathrm{~cm} \mathrm{AK}$ gap. For the histograms modeling the PD time dependences, sequential 5-ns-duration LSP electron-beam distributions are incident on the diode hardware in ITS, and the resulting incremental far-field photon spectra angular
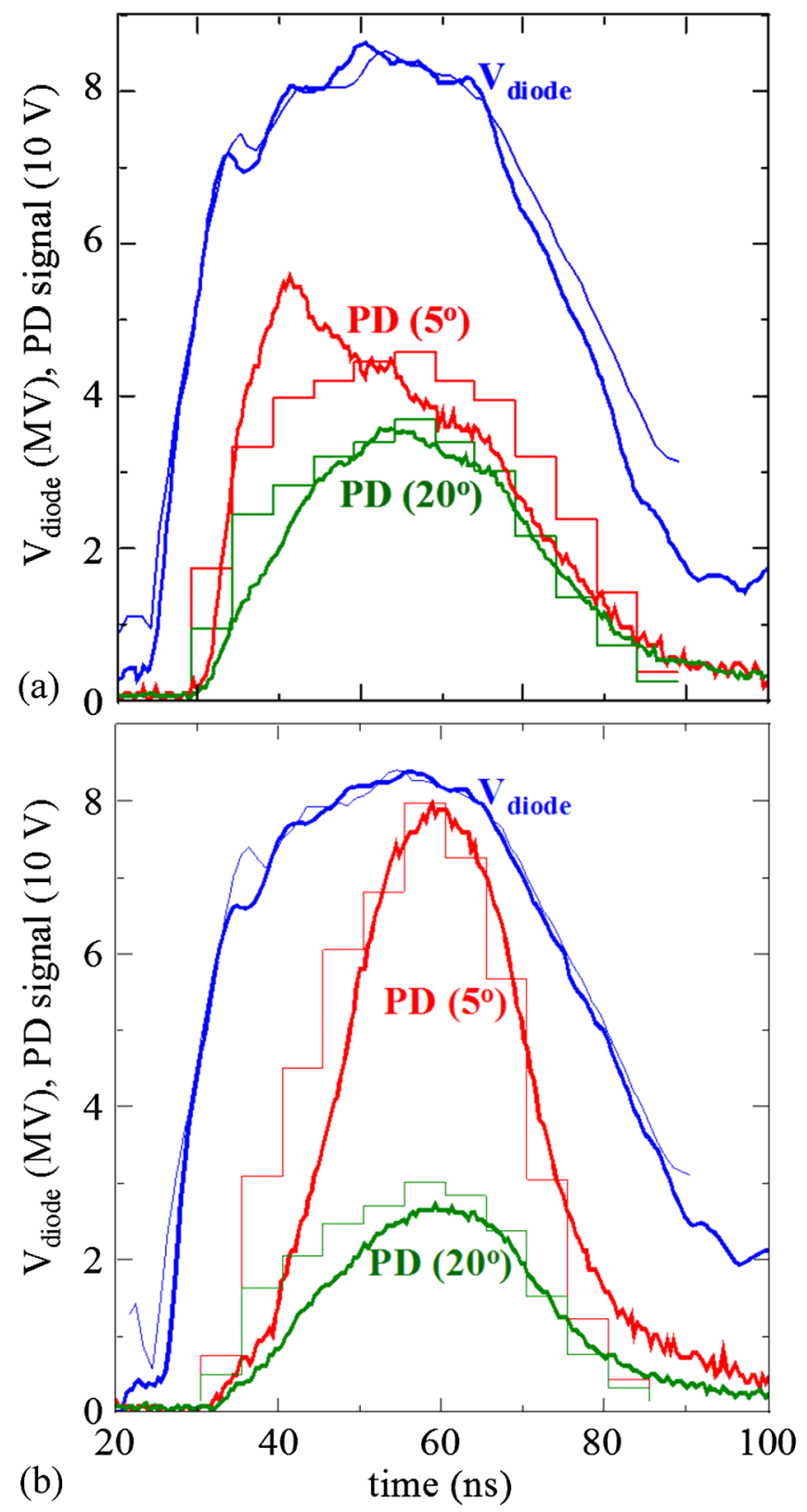

FIG. 11. (a) Time-dependent photodiode measurements and LSP/ITS computations for a $23 \mathrm{~cm} \mathrm{AK} \mathrm{gap.} \mathrm{Measured} \mathrm{diode}$ voltage and photodiode traces for shot 1330 are bold curves. The finer voltage curves and PD histograms are computed from LSP/ ITS. (b) Time-dependent photodiode measurements and LSP/ITS computations for a $40 \mathrm{~cm}$ AK gap. The same quantities are plotted for shot 1295 as in Fig. 11(a). 
distributions are used to compute the dose in plastic scintillators at two angles: $5^{\circ}$ and $20^{\circ}$. The computed $5^{\circ}$ PD signals are then adjusted in amplitude to fit the measured $5^{\circ}$ signals. The $20^{\circ}$ signal amplitude is scaled with the $5^{\circ}$ signal.

The measured $5^{\circ}$ PD signal in Fig. 11(a) shows an early spike not observed in the measured $20^{\circ}$ signal or in the simulations. This spike is attributed to the time when the full electron beam first illuminates the converter as it sweeps radially inward on the current rise. At this time, electrons impact the converter most closely to normal incidence, as indicated by the factor of 2 smaller $20^{\circ}$ signal. The subsequent reduction in the $5^{\circ}$ signal is then associated with increasing electron incidence angles with further radial compression. Later in time, the measured $5^{\circ}$ PD signal is close to that at $20^{\circ}$, suggesting that electronincidence angles are tens of degrees (top of Fig. 4). The early spike behavior of Fig. 11(a) is not observed in the simulations. It is believed that this is because the $2 \mathrm{D}$ simulations predict lower electron losses than experienced in the experiment. For the $23 \mathrm{~cm}$ gap simulations, the electron beam losses to the wall are negligible and the full electron beam impacts the converter well before voltage maximum, so that the dose rate is smoothly varying in time.

For the $40 \mathrm{~cm}$ gap PD signals of Fig. 11(b), beam losses to the anode insert dominate the behavior as described by Fig. 3. As with the converter current, the $\mathrm{x}$ rays predicted by LSP/ITS lead the measured signals because of superior 2D electron confinement. At peak voltage, both measured and calculated PD signals at $20^{\circ}$ are about a factor of 3 smaller than those at $5^{\circ}$, showing that electron impact angles on the converter for the $40 \mathrm{~cm}$ gap are much smaller than for the $23 \mathrm{~cm}$ gap.

The angular dose distributions from TLD and photodiode diagnostics measured the x-ray divergence at low photon energies, which depended on the electron angles of incidence onto the converter. For optimal on-axis far-field photofission yield, the time-integrated incidence angles for electrons above $5 \mathrm{MeV}$ should strike the converter normal to its surface. Figure 12(a) shows the LSP-simulated angular distribution of electrons striking the converter for several AK-gap configurations. The resulting bremsstrahlung distributions were transported to the DU plate, convolved with the photofission cross section, and integrated over photon energy to predict the off-axis far-field photofission yields as a function of DU-plate angle with respect to the axis of symmetry. These photofission yields are shown in Fig. 12(b) for the electron distributions of Fig. 12(a). The improvement in forward-directed electrons (i.e., small incidence angles) is clearly shown since increasing the AK gap from 23 to $40 \mathrm{~cm}$ resulted in a more highly directed bremsstrahlung beam, especially at high photon energies. This improvement translated into photofission yields that were more forward directed in angle and higher on axis. A slight reduction in yield
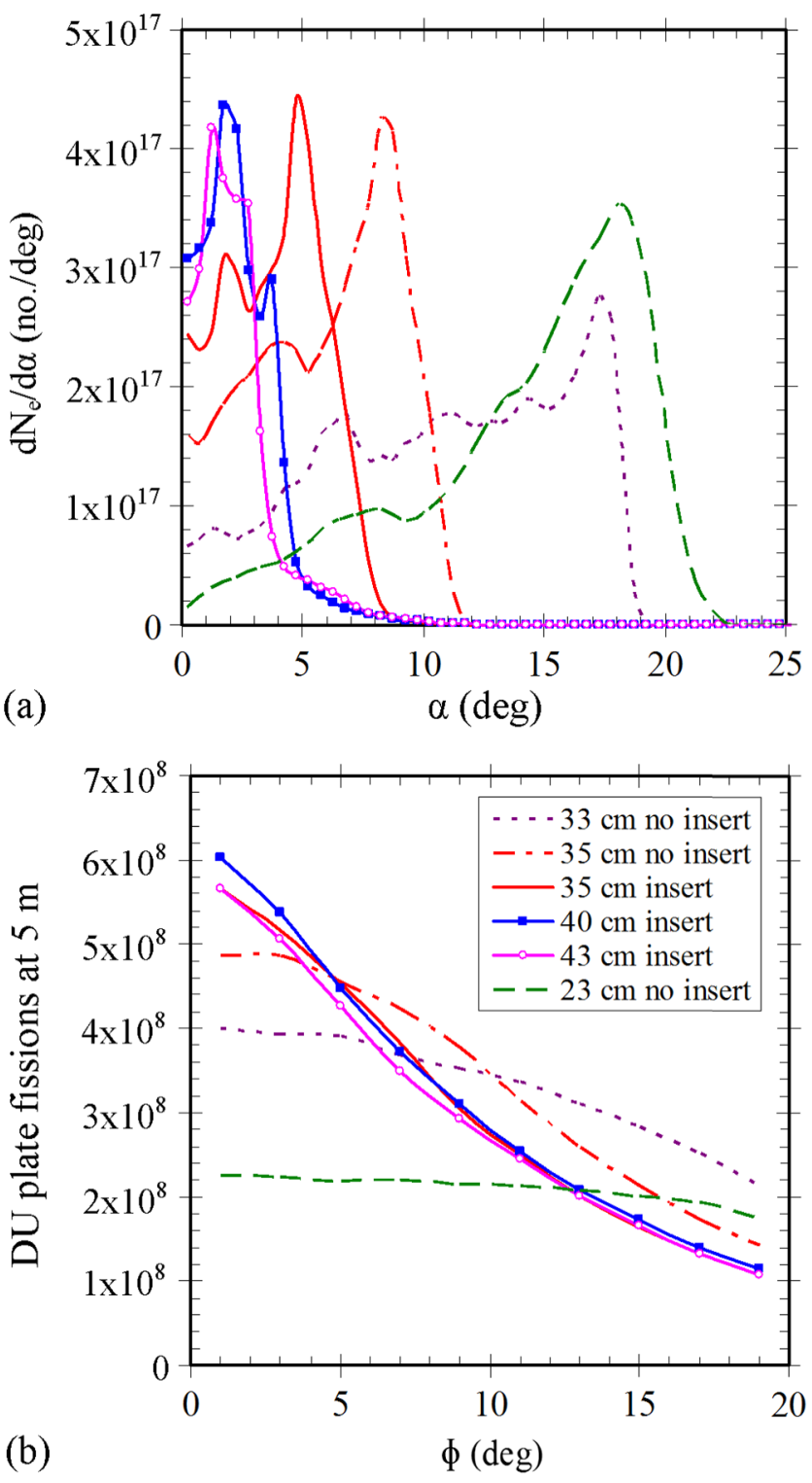

FIG. 12. (a) Distributions of time-integrated electron incidence angles on the converter for different $\mathrm{AK}$ gaps as computed by LSP. Here, $\alpha$ is the momentum-space angle measured from the axis of symmetry. Curve labels are as in Fig. 12(b). (b) Variation of fission yield in the DU plate $5 \mathrm{~m}$ from the converter vs the angle the plate is displaced from the axis of symmetry. Here, $\Phi$ is the configuration-space angle measured from the axis of symmetry to a normal through the center of the plate.

between 40 and $43 \mathrm{~cm}$ is predicted because the $\mathrm{x}$-ray divergence does not narrow significantly for the narrower electron angular distribution. However, electron losses to the insert and wall continue to increase for larger gaps, causing the reduced yield at $43 \mathrm{~cm}$. The benefit of using the anode insert is shown by comparing the simulated $35 \mathrm{~cm}$ cases with and without it. Though the electron-beam losses are greater with the insert, the more-forward-directed electrons produce a higher far-field fission yield on axis. 
The He-3 detector was used to measure the delayed neutrons emitted from the DU plate as a result of induced photofissions from the bremsstrahlung beam. The measured delayed neutron counts are summarized in Fig. 13 as a function of AK gap for the on-axis and off-axis DU plate positions and are shown to agree well with the counts predicted by LSP/ITS. The neutron counts were integrated between $100 \mathrm{~ms}$ and $100 \mathrm{~s}$ after the shot. The induced neutron background measured by the He-3 detector lasted less than $5 \mathrm{~ms}$ (determined by shots without the DU present) and was therefore excluded by starting the count at $100 \mathrm{~ms}$. The passive background rate was 0.022 counts/s, so the signal-to-background ratio was greater than 700 for all cases. The uncertainties in the experimental data reflect the shot-to-shot variations. The predicted neutron counts from the He-3 detector are computed from the yield curves of Fig. 12(b) and two simulations using the Monte Carlo radiation transport code MCNPX [35]. The first MCNPX computation used the Mercury bremsstrahlung spectrum incident on the DU plate to compute the delayed neutron energy spectrum at the location of the He-3 detector, including neutrons reflected from the Mercury test cell environment. The second used this neutron spectrum to determine the number of $\mathrm{He}-3$ counts for a given neutron fluence incident on the detector. These computations result in $8.9 \times 10^{4}$ fissions in the

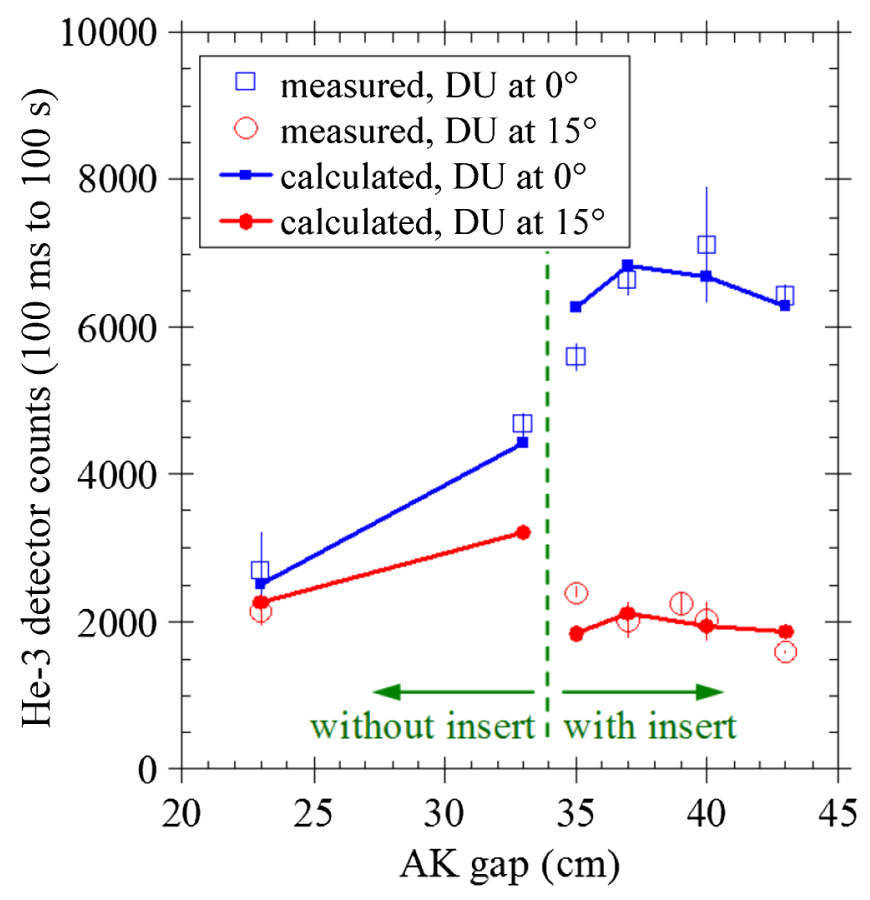

FIG. 13. Measured He-3 counts between $100 \mathrm{~ms}$ and $100 \mathrm{~s}$ for a detector $1 \mathrm{~m}$ from the DU plate on axis (squares) and $15^{\circ}$ off axis (circles) as a function of AK gap. Error bars reflect shot-to-shot variations for each geometry. The curves are from LSP/ITS predictions of Fig. 12(b) and a conversion to He-3 counts described in the text.
DU plate for each He-3 count when the detector is $1 \mathrm{~m}$ from the plate.

The overall increase in forward-directed photofission yield with the larger AK gaps was correctly reproduced, as was the decrease in photofission yield for the largest AK gaps resulting from current loss to the anode insert. The simulations did differ slightly from measurements in the predicted optimum gap for photofission yield at $0^{\circ}$. This difference is within experimental uncertainties and the limitations of 2D current-loss modeling. It is worth noting that the absolute-value comparisons of neutron-count measurements and modeling do not show the systematic differences observed with the dose (Fig. 10). The dose discrepancy has been associated with differences in measured and modeled converter currents during the rising portion of the pulse (Fig. 6). However, the fission yield depends only on the highest-energy photons in the bremsstrahlung spectrum, and the computed fission rate time dependence is more strongly peaked around peak voltage than the dose rate, with few neutrons produced during the times when the computed and measured converter currents are different.

The off-axis He-3 count predictions based on Fig. 12(b) also agree well with measurements. Taken with the on-axis results, delayed-fission-neutron counting has been shown to be a valuable new quantitative diagnostic for energetic $(>5 \mathrm{MeV})$ photons. Moreover, because of the narrow intrinsic radiation cone of these photons, the photofission angular dependence is a more sensitive measure of electron incidence angles onto the converter than is the dose, important for understanding this class of radiation source in the far field.

\section{SUMMARY AND FUTURE WORK}

The IPAD technique is based on inducing a large burst of fissions, and therefore requires an intense source of probing radiation. A high-power vacuum diode was designed and coupled to the Mercury pulsed power generator with the goal of maximizing the far field photofission yield in a depleted uranium object by varying the anode-cathode gap. This experiment was diagnosed in detail to measure the electron beam dynamics, the dose map of the bremsstrahlung, and the induced photofissions as a function of angle in the far field. The experiment was successfully modeled using the simulation codes LSP and ITS with excellent agreement between the simulation predictions and the measured angular fission yield. The tradeoff between electron angle of incidence onto the converter and beam current losses to an indented outer conductor was found to produce a local maximum in on-axis photofission with a $40 \mathrm{~cm}$ anode-cathode gap.

Under the optimum conditions, most of the useful bremsstrahlung radiation is produced near the outside of the 20-cm-diameter converter. For improved fissilematerial detection in the field, a smaller-diameter electron 
beam would be easier to collimate. To this end, ongoing research is investigating the self-magnetic lens diode. This new diode may produce a more forward-directed electron beam while mitigating current losses to the outer conductor by using the beam's self-induced magnetic field to straighten the electron-incidence angles at smaller radius [37]. This straightening at smaller beam radius would permit the use of smaller, lighter beam-collimation hardware.

\section{ACKNOWLEDGMENTS}

The authors thank Tony Culver, Derric Featherstone, and Aaron Miller for their hard work and dedication in operating the Mercury generator and for reloading the numerous TLDs during the experiment. The authors also thank the DOD High Performance Computing Modernization Program at the Naval Research Laboratory for a grant of computer time. This work was supported by the U.S. Office of Naval Research.

[1] R. C. Runkle, D. L. Chichester, and S. J. Thompson, Nucl. Instrum. Methods Phys. Res., Sect. A 663, 75 (2012).

[2] S. B. Swanekamp, J. P. Apruzese, R. J. Commisso, D. Mosher, and J. W. Schumer, IEEE Trans. Nucl. Sci. 58, 2047 (2011).

[3] C. Hill, J. O’Malley, M. Ellis, P. Mistry, R. Maddock, J. Precious, J. C. Zier, S. L. Jackson, A. L. Hutcheson, L. Mitchell, and B. F. Phlips, in Proceedings of the 2012 IEEE Nuclear Science Symposium and Medical Imaging Conference, Anaheim, CA, 2012, (Institute of Electrical and Electronic Engineers, Piscataway, NJ, 2013) pp. 1424-1429.

[4] C. Hill, J. O’Malley, P. Martin, K. Marshall, R. Maddock, J. Threadgold, R. J. Commisso, S. L. Jackson, J. W. Schumer, B. F. Phlips, P. F. Ottinger, D. Mosher, J. P. Apruzese, F. C. Young, and J. Davis, in Proceedings of the 2012 IEEE Nuclear Science Symposium and Medical Imaging Conference, Anaheim, CA, 2012, (Institute of Electrical and Electronic Engineers, Piscataway, NJ, 2013) pp. 732-737.

[5] R. S. Woolf, B. F. Phlips, A. L. Hutcheson, E. A. Wulf, J. C. Zier, S. L. Jackson, R. J. Commisso, J. W. Schumer, C. Clemett, J. O’Malley, C. Hill, R. Maddock, P. Martin, and J. Threadgold, in Proceedings of the 2013 IEEE Nuclear Science Symposium and Medical Imaging Conference, Seoul, Korea, 2013.

[6] M. Ellis, L. Mitchell, S. L. Jackson, J. C. Zier, J. O’Malley, J. R. Threadgold, C. D. Clemett, A. Thandi, and P. N. Martin, in Proceedings of the 2012 IEEE Nuclear Science Symposium and Medical Imaging Conference, Anaheim, $C A, 2012$, (Institute of Electrical and Electronic Engineers, Piscataway, NJ, 2013) pp. 11-17.

[7] S. B. Swanekamp, J. P. Apruzese, R. J. Commisso, S. L. Jackson, D. Mosher, J. W. Schumer, B. V. Weber, and J. C. Zier, in Proceedings of 2012 IEEE Nuclear Science Symposium and Medical Imaging Conference, Anaheim,
CA, 2012 (Institute of Electrical and Electronic Engineers, Piscataway, NJ, 2013).

[8] R. J. Allen, C. L. Berry, R. J. Commisso, G. Cooperstein, R. Fisher, D. D. Hinshelwood, T. A. Holt, A. T. Miller, D. P. Murphy, J. M. Neri, P. F. Ottinger, D. G. Phipps, J. W. Schumer, S. J. Stephanakis, S. B. Swanekamp, F. C. Young, D. L. Johnson, and I. Smith, in Proceedings of the 15th IEEE International Pulsed Power Conference, Monterey, CA, 2005, (Omnipress, Madison, WI, 2005) pp. 318-321.

[9] R. J. Allen, R. J. Commisso, G. Cooperstein, P. F. Ottinger, and J.W. Schumer, in Proceedings of the 18th IEEE International Pulsed Power Conference, Chicago, IL, 2011, (Omnipress, Madison, WI, 2011) pp. 1036-1041.

[10] J. C. Zier, D. Mosher, S. L. Jackson, A. S. Richardson, R. J. Commisso, G. Cooperstein, D. D. Hinshelwood, D. P. Murphy, P. F. Ottinger, J. W. Schumer, and S. B. Swanekamp, in Proceedings of the IEEE Pulsed Power and Plasma Science Conference, San Francisco, CA, 2013 (Omnipress, Madison, WI, 2013).

[11] LSP is a product of Mission Research Corporation, Albuquerque, NM, 87110.

[12] J. Halbleib, R. Kensek, and G. Valdez, IEEE Trans. Nucl. Sci. 39, 1025 (1992).

[13] J. Maenchen, G. Cooperstein, J. O’Malley, and I. Smith, Proc. IEEE 92, 1021 (2004).

[14] G. Cooperstein, J. R. Boller, R. J. Commisso, D. D. Hinshelwood, D. Mosher, P. F. Ottinger, J. W. Schumer, S. J. Stephanakis, S. B. Swanekamp, B. V. Weber, and F. C. Young, Phys. Plasmas 8, 4618 (2001).

[15] B. V. Oliver, D. Short, G. Cooper, J. McLean, and J. O’Malley, IEEE Trans. Plasma Sci. 33, 704 (2005).

[16] D. D. Hinshelwood, R. J. Allen, R. J. Commisso, G. Cooperstein, B. M. Huhman, D. Mosher, D. P. Murphy, P. F. Ottinger, J. W. Schumer, S. B. Swanekamp, S. J. Stephanakis, B. V. Weber, F. C. Young, I. Crotch, J. O'Malley, and J.R. Threadgold, IEEE Trans. Plasma Sci. 35, 565 (2007).

[17] T. W. L. Sanford, J. W. Poukey, R. C. Mock, and D. R. Welch, J. Appl. Phys. 71, 472 (1992).

[18] T. W. L. Sanford, J. A. Halbleib, J. W. Poukey, D. E. Beutler, G. A. Carlson, G. T. Baldwin, T. Sheridan, R. Mock, R. S. Klingler, and D. P. Knott, IEEE Trans. Nucl. Sci. 36, 1931 (1989).

[19] T. W. L. Sanford, Phys. Fluids B 3, 2387 (1991).

[20] T. W. L. Sanford, J. A. Halbleib, W. H. McAtee, K. A. Mikkelson, R. C. Mock, and J. W. Poukey, J. Appl. Phys. 69, 7283 (1991).

[21] T. W. L. Sanford and R. C. Mock, IEEE Trans. Nucl. Sci. 39, 2060 (1992).

[22] D. D. Hinshelwood, R. J. Allen, J. P. Apruzese, R. J. Commisso, G. Cooperstein, S. L. Jackson, and D. Mosher, in Proceedings of the 18th IEEE International Pulsed Power Conference, Chicago, IL, 2011, (Omnipress, Madison, WI, 2011) pp. 1428-1435.

[23] T. W. L. Sanford, J. A. Halbleib, J. W. Poukey, A. L. Pregenzer, R. C. Pate, C. E. Heath, R. Mock, G. A. Mastin, D. C. Ghiglia, T. J. Roemer, P. W. Spence, and G. A. Proulx, J. Appl. Phys. 66, 10 (1989). 
[24] A. E. Blaugrund, G. Cooperstein, and S. A. Goldstein, Phys. Fluids 20, 1185 (1977).

[25] J. P. VanDevender, J. Appl. Phys. 50, 3928 (1979).

[26] C. W. Mendel and S. E. Rosenthal, Phys. Plasmas 2, 1332 (1995).

[27] C. W. Mendel, J. Appl. Phys. 50, 3830 (1979).

[28] C. W. Mendel and S. E. Rosenthal, Phys. Plasmas 3, 4207 (1996).

[29] P. F. Ottinger and J. W. Schumer, Phys. Plasmas 13, 063109 (2006).

[30] T. W. L. Sanford, J. W. Poukey, T. P. Wright, J. Bailey, C. E. Heath, R. Mock, P. W. Spence, J. Fockler, and H. Kishi, J. Appl. Phys. 63, 681 (1988).

[31] J. T. Caldwell, E. J. Dowdy, B. L. Berman, R. A. Alvarez, and P. Meyer, Phys. Rev. C 21, 1215 (1980).

[32] B. V. Weber, R. J. Commisso, S. L. Jackson, D. P. Murphy, S. B. Swanekamp, and J.C. Zier, in Proceedings of the IEEE Pulsed Power and Plasma Science Conference, San Francisco, CA, 2013.
[33] D. P. Murphy, S. L. Jackson, J. C. Zier, D. D. Hinshelwood, R. J. Commisso, A. S. Richardson, J. W. Schumer, S. B. Swanekamp, D. Mosher, G. Cooperstein, and P. F. Ottinger, in Proceedings of the IEEE Pulsed Power and Plasma Science Conference, San Francisco, CA, 2013.

[34] T. W. L. Sanford, J. W. Poukey, J. A. Halbleib, R. C. Mock, and W. H. McAtee, J. Appl. Phys. 73, 2004 (1993).

[35] D. Mosher, J. W. Schumer, J. P. Apruzese, R. J. Commisso, D. D. Hinshelwood, S. L. Jackson, D. P. Murphy, and S. B. Swanekamp, in Proceedings of the 18th IEEE International Pulsed Power Conference, Chicago, IL, 2011, pp. 1436-1441.

[36] T. W. L. Sanford, J. A. Halbleib, J. W. Poukey, C. E. Heath, and R. Mock, Nucl. Instrum. Methods Phys. Res., Sect. B 34, 347 (1988).

[37] S. B. Swanekamp, G. Cooperstein, D. D. Hinshelwood, D. Mosher, P. F. Ottinger, J. W. Schumer, B. V. Weber, and J. C. Zier, in Proceedings of the IEEE Pulsed Power and Plasma Science Conference, San Francisco, CA, 2013. 